\title{
Glycogen storage disease due to phosphoglycerate mutase deficiency
}

INSERM

\section{Source}

INSERM. (1999). Orphanet: an online rare disease and orphan drug data base. Glycogen storage disease due to phosphoglycerate mutase deficiency. ORPHA:97234

Muscle phosphoglycerate mutase deficiency (PGAMD) is a metabolic myopathy characterised by exercise-induced cramp, myog lobinuria, and presence of tubular aggregates in the muscle biopsy. Serum creatine kinase (CK) levels are increased between episodes of myog lobinuria. Less than 50 cases have been described so far. The disease is due to an anomaly in one of the last steps of glycolysis. The enzymatic defect in PGAMD is caused by mutations in the CDNA coding for the M-isoform of PGAM. Residual PGAM activity in the muscles of patients (2\%-6\%) is due to activity of the Bisoform. Transmission is autosomal recessive. Differential diagnosis includes muscle phosphorylase deficiency (McArdle disease) and phosphofructokinase deficiency (PFKD) (see these terms). 